\title{
Impact of Dynamic Traffic on Vehicle-to-Vehicle Visible Light Communication Systems
}

Alsalami, Farah Mahdi ; Haas, Olivier; Al-Kinani, Ahmed; Wang, Cheng-Xiang; Ahmad, Zahir; Rajbhandari, Sujan

\section{IEEE Systems Journal}

DOI:

https://doi.org/10.1109/JSYST.2021.3100257

Published: 01/09/2022

Peer reviewed version

Cyswllt i'r cyhoeddiad / Link to publication

Dyfyniad o'r fersiwn a gyhoeddwyd / Citation for published version (APA):

Alsalami, F. M., Haas, O., Al-Kinani, A., Wang, C-X., Ahmad, Z., \& Rajbhandari, S. (2022).

Impact of Dynamic Traffic on Vehicle-to-Vehicle Visible Light Communication Systems. IEEE Systems Journal, 16(3), 3512-3521. https://doi.org/10.1109/JSYST.2021.3100257

\footnotetext{
Hawliau Cyffredinol / General rights

Copyright and moral rights for the publications made accessible in the public portal are retained by the authors and/or other copyright owners and it is a condition of accessing publications that users recognise and abide by the legal requirements associated with these rights. study or research

- Users may download and print one copy of any publication from the public portal for the purpose of private

- You may not further distribute the material or use it for any profit-making activity or commercial gain

- You may freely distribute the URL identifying the publication in the public portal?
}

Take down policy

If you believe that this document breaches copyright please contact us providing details, and we will remove access to the work immediately and investigate your claim. 


\title{
Impact of Dynamic Traffic on Vehicle-to-Vehicle Visible Light Communication Systems
}

\author{
Farah Mahdi Alsalami, Student Member, IEEE, Olivier C.L. Haas, Senior Member, IEEE, Ahmed \\ Al-Kinani, Cheng-Xiang Wang, Fellow, IEEE, Zahir Ahmad, and Sujan Rajbhandari, Senior Member, IEEE
}

\begin{abstract}
This paper studies the impact of dynamic vehicular traffic density on the signal-to-noise-ratio (SNR) and the associated bit-error-rate (BER) performance of vehicle-to-vehicle visible light communication (V2V-VLC) systems. The study uses traffic data from the M42 and M6 motorways in the UK to investigate the probability of co-existence of other vehicles in the adjacent lanes which induce interference and act as potential reflectors. The results show that the probability of co-existence of other vehicles in the adjacent lanes is lane-independent and it increases during the rush hours to $90 \%$, while it decays to less than $10 \%$ during the off-peak and early morning hours. The inter-vehicular distance and the BER performance vary widely between different lanes and different periods of the day. The results also show that the BER performance of V2V-VLC system with non-line-of-sight (NLOS) component and with line-of-sight (LOS) component are comparable at rush hours. However, high BER values are predicted during the off-peak hours for NLOS components of the channel.
\end{abstract}

Index Terms-dynamic traffic conditions, dynamic vehicular traffic density, Vehicular communication channel model, Vehicular communications, visible light communication (VLC).

\section{INTRODUCTION}

$\mathbf{R}$ OAD accidents, mostly caused by human errors, are one of the main causes of accidental death in the world [1], [2]. Traffic management technologies and systems exploiting Intelligent transport system (ITS) aim to reduce human errors. Based on ITS, many innovative systems have been proposed, such as vehicle-to-vehicle (V2V), vehicle-toinfrastructure (V2I), vehicle-to-pedestrian (V2P), and most recently vehicle-to-everything (V2X) communications [2]-[5]. Such communication aims to improve drivers' awareness and enable connected and autonomous safety features on vehicles.

The literature suggests that wireless communication technologies, such as dedicated short-range communications (DSRC), IEEE 802.11p, LTE-V2V, 5G, and more recently 6G can enable connectivity between vehicles and with the infrastructure [4], [6]. The allocated bandwidth of the suggested

F. M. Alsalami and O.C.L. Haas are with the Research Institute for Future Transport and Cities, Coventry University, Coventry CV1 5FB, UK. (E-mails: alsallaf@uni.coventry.ac.uk and o.haas@coventry.ac.uk).

A. Al-Kinani is with MMX Communications Services Limited, Birmingham B90 4SH, U.K (E-mails: aalkinani@mmxcomms.com).

C.-X. Wang is with the National Mobile Communications Research Laboratory, School of Information Science and Engineering, Southeast University, Nanjing 210096, China, and also with the Purple Mountain Laboratories, Nanjing 211111, China (E-mails: chxwang@seu.edu.cn).

Z. Ahmad is with the Faculty of Engineering, Environment and Computing, Coventry University, Coventry CV1 5FB, UK. (E-mails: ab7175@ coventry.ac.uk).

S. Rajbhandari (corresponding author) is with the school of Computer Science and Electronic Engineering, Bangor University, Bangor, LL57 1UT, UK (email: s.rajbhandari@bangor.ac.uk) technologies, which use the radio frequency (RF) spectrum, is limited [7]. Hence, it is expected for this technology to suffer from interference and bandwidth scarcity [7]-[9]. Visible light communication (VLC) is proposed as a complementary technology to reduce the bandwidth congestion as well as free up the RF spectrum for critical applications [3], [8]-[10].

VLC utilizes existing street-lights, traffic lights, vehicles' headlights, and vehicles' taillights to transmit traffic data between vehicles and interact with the infrastructure. In addition, due to the directional nature of light sources, VLC incurs less interference compared to RF [2], [9], [11].

The dynamic nature of the vehicular communication system results in a high probability for VLC links to be interrupted due to interference and reflection from the adjacent vehicles in the surrounding lanes. Interference from neighbouring artificial light sources of the adjacent vehicles increases the shot noise [2], [11], [12]. Furthermore, the reflected light from the adjacent vehicles in the neighbouring lanes disperses the received signal pulses and consequently introduces the intersymbol interference (ISI) problem which limits data rates. Hence, it is important to investigate the statistics of the existence of other vehicles in the adjacent lanes. In the following section, we provide related works that discussed the impact of interference and reflection from natural and artificial sources on vehicular visible light communication (VVLC) systems, which include V2V, V2I, V2P, and V2X communications.

\section{A. Related works}

The impact of interference from natural optical sources has been investigated in [13]-[16]. The impact of the sunlight interference on VLC system performance studied in [13] indicated that there is more than $20 \mathrm{~dB}$ variation in background noise powers between the lower levels in the early morning and its peak during noon. Therefore, a diversity receiver with the selective combining technique was proposed which improved signal-to-noise-ratio (SNR) by $5 \mathrm{~dB}$. The study in [14] evaluated the degradation in SNR, data rate, and BER performance of VLC systems under the presence of sunlight in different locations. The study estimated the solar irradiance variations during different hours of the day and different seasons. The results showed that VLC link with a data rate of $1 \mathrm{Gbps}$ can be achieved in presence of sunlight without optical filter whilst blue filters offer $6.47 \mathrm{~dB}$ improvement in SNR. Likewise, [15] shows that an optical filter can block the out-of-band ambient lights with an incident angle greater than $30^{\circ}$. The study in [16] accounted for sunlight irregularities and identified the maximum and minimum solar 
irradiation for every day of the year. The results showed that maximum radiation reduces the transmission distance by $5 \mathrm{~m}$ for binary pulse amplitude modulation scheme.

However, only limited research activities investigated the impact of interference from artificial light sources in VVLC systems. The work reported in [17] considered the impact of interference due to vehicles in the adjacent lanes on the performance of VVLC for Platoon scenario. The results showed that the performance decays when the rate of arrival of vehicles in the adjacent lanes increases. The work in [9] highlighted the impact of interference at high traffic density in the VLC and RF systems. According to [9], VLC has a low interference level and limited transmission range. Therefore, the study suggested a hybrid VLC/RF-enabled Vehicular Adhoc Network (VANET). VLC technology was utilized due to broad bandwidth, high power efficiency, and relatively low reflection. RF was utilized to support longer transmission ranges. The work in [8] considered VLC technology to tackle the interference and delay in RF-based V2V systems under dense traffic conditions. The study proposed VLC for dual functions of communications and distance estimation.

A geometry-based analytical method to study V2V visible light communication (V2V-VLC) channel was considered in [18], [19]. The work in [18] investigated the effect of the light reflection from the road surface and road dirt. However, the study utilized a tungsten-halogen headlight beam pattern, which may not be applicable for V2V-VLC systems that use light-emitting diode (LED) light sources. The results showed that wet roads increase the received power of the reflection and hence increase the transmission distance. The study in [18] was extended in [19] to increase the transmission data rate of V2V-VLC link using a multiple-input multipleoutput (MIMO) system. The $2 \times 2$ MIMO system achieved a data rate of $4 \mathrm{Mbps}$ for a transmission distance of $40 \mathrm{~m}$. These studies, however, did not consider the reflection from the adjacent vehicles which can be significant, particularly in dense traffic conditions.

A two-dimensional (2D) geometry-based stochastic model (GBSM) with two rings and an ellipse to investigate the V2VVLC channels was proposed in [3]. The study considered lineof-sight (LOS) and reflected power from surrounding vehicles and roadsides in dynamic V2V-VLC environments. The results showed that the received power due to the reflection and scattering from roadsides and vehicles in the dynamic V2VVLC channel is less than the received power for the LOS component by at least two orders of magnitude. The study in [3] was extended in [20] to consider the asymmetrical radiation pattern of vehicles' headlights. The study used a threedimensional (3D) GBSM with two spheres and an ellipsoid to model the V2V-VLC channel. The results highlighted the importance of considering the $3 \mathrm{D}$ radiation pattern of the vehicle's headlights. Likewise, the study in [21], used the GBSM to study the received power of the direct LOS and reflections from surrounding vehicles in dynamic V2V-VLC channel. The study showed that the variation in the relative speed of vehicles impacts the reflected power more than the LOS power. The direction of the vehicle's motion affects the received power from the LOS component more than the received power of the reflection component.

VVLC systems are affected by multiple variables including radiation pattern, weather conditions, interference from artificial and natural light sources, traffic conditions, coating and colour of cars as well as the condition of road surfaces (wet or dry). However, these variables are independent. Hence, a universal model that considers the impact of these variables can be found by combining the impact of independent variables. The effect of the radiation pattern on VVLC was considered in [22], where analytical models that can describe the radiation pattern of headlamp from different manufacturers and designs were proposed. The impact of different weather conditions and specular reflections from surrounding vehicles and road was considered in [23]. The study showed that the propagation path loss due to the inter-vehicle distance variation is significantly higher than the attenuation due to adverse weather condition (e.g. dense fog). In addition, the reflections from street furniture have a marginal impact compared to the reflection from vehicles due to the modest reflectivity of other surfaces. Therefore, in this study, we focus on the reflection from surrounding vehicles in the adjacent lanes and dynamic behaviours of the VVLC channel due to traffic conditions during different times of the day.

The previous studies identified the impact of the interference and reflection on VVLC channel. However, none of these studies investigated the statistics of the existence of other vehicles in the adjacent lanes, which can significantly affect the levels of interference and reflection.

\section{B. Motivation and Original Contribution}

Surrounding vehicles with a body characterised by a relatively high reflectivity are the closest reflective objects to the V2V-VLC link. Therefore, they are potential candidates to reflect the communication light rays. In addition, interference from adjacent vehicles light sources increases the shot noise [11], [12], [24]. However, due to the dynamic nature of traffic, which varies during different periods of the day, the presence of the adjacent vehicles changes with the traffic flow. Therefore, our motivation is to evaluate the effect of interference and reflection from the surrounding vehicles in the adjacent lanes and study their impact on V2V-VLC performance during different times of the day based on real traffic measurement data.

The novelty and original contributions of this work are:

- Use traffic measurements to estimate the probability of co-existence of other vehicles in the adjacent lanes. This is expected to be an important factor to examine the performance of vehicular communication systems regardless of the communication technologies in use.

- Consider the radiation pattern model for low-beam and high-beam headlights to study the LOS and non-line-ofsight (NLOS) components of the V2V-VLC channel.

- Study the impact of dynamic vehicular traffic density on V2V-VLC channel SNR and BER performance in different Lanes.

The rest of the paper is organized as follows. The V2V-VLC link performance is presented in Section II. The V2V-VLC 


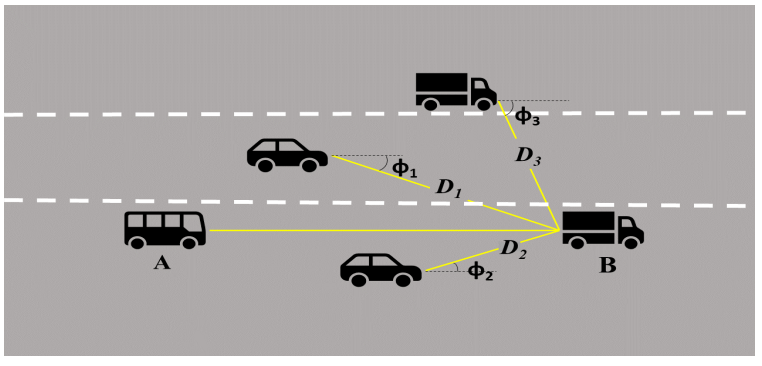

(a)

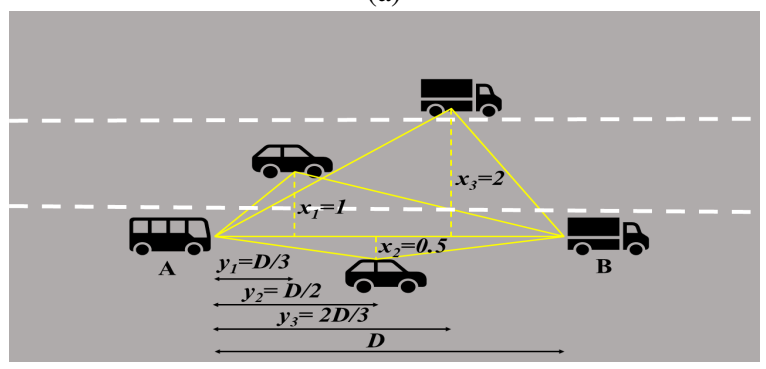

(b)

Fig. 1: A typical communication link between two vehicles travelling on a three-lane motorway and co-existence of other vehicles in the adjacent lanes which act as a) interference sources and b) reflectors.

channel gain is explained in Section III. The probability of coexistence of other vehicles in the adjacent lanes is presented in Section IV. Numerical results are discussed in Section V. Finally, conclusions are drawn in Section VI.

\section{THE V2V-VLC LINK PERFORMANCE}

The proposed V2V-VLC link scenario is shown in Fig. 1. The transmitter vehicle $\mathrm{A}$ and the receiver vehicle $\mathrm{B}$ drive on a motorway in the presence of other vehicles in the adjacent lanes. The surrounding vehicles can act as ambient light interference sources and/or as potential reflectors.

If $x(t)$ is the transmitted optical signal, then the received signal $y(t)$ is given by [25]

$$
y(t)=\gamma h(t) \circledast x(t)+n(t) .
$$

Here $\circledast$ refers to convolution operation, $\gamma$ denotes the receiver's responsivity, $h(t)$ is the channel impulse response and $n(t)$ is the additive white Gaussian noise (AWGN) with zero mean and variance $\sigma_{T}^{2}$.

The AWGN is caused by thermal and ambient noises. The total noise variance $\sigma_{T}^{2}$ is given by [8], [18]

$$
\sigma_{T}^{2}=\sigma_{\text {shot }}^{2}+\sigma_{t h}^{2}
$$

where $\sigma_{\text {shot }}^{2}$ and $\sigma_{\text {th }}^{2}$ are the shot and thermal noise variances, respectively.

The thermal noise variance is given by [11]

$\sigma_{t h}^{2}=\frac{8 \pi K_{k} T_{k} C_{p d} A_{r} I_{2} B_{w}^{2}}{G_{\nu}}+\frac{16 \pi^{2} K_{k} T_{k} \Gamma C_{p d}^{2} A_{r}^{2} I_{3} B_{w}^{3}}{g_{m}}$

where $K_{k}$ denotes the Boltzmann's constant, $T_{k}$ is the absolute temperature, $C_{p d}$ refers to the fixed capacitance of a photodiode per unit area, $A_{r}$ is the active area of the receiver, $I_{2}$ and $I_{3}$ are the noise bandwidth factors, $B_{w}$ is the noise bandwidth,
$G_{\nu}$ indicates the open-loop voltage gain, $\Gamma$ is the FET channel noise factor and $g_{m}$ denotes FET transconductance.

The background daylight and the artificial light (such as the lights from the adjacent vehicles) are the main sources of ambient noise in V2V-VLC systems [8], [18]. However, daylight is the dominant source of ambient noise [18], which peaks only at morning hours [13]. During the night, the artificial light sources of the adjacent vehicles are the main sources of ambient noise. The incident luminous flux of the ambient noise induces a shot noise, which is given by [13]

$$
\sigma_{\text {shot }}^{2}=2 q\left(P_{R}+L P_{I}\right) \gamma B_{w}+2 q I_{b g} I_{2} B_{w}
$$

where $q$ is the electron charge, $P_{R}$ is the received power, $L$ describes the probability of co-existence of other vehicles in the adjacent lanes, $P_{I}$ is the interference power from vehicles in adjacent lanes as illustrated in Fig. 1a, $I_{b g}$ is the current induced by the ambient light sources.

The received power $P_{R}$ in V2V-VLC is a linear superposition of the power from LOS and NLOS paths. The received power $P_{R}$ is calculated from the transmitted power $P_{T}$ as [11]

$$
P_{R}=P_{T} H(0)
$$

where $H(0)$ is the $\mathrm{DC}$ channel gain given as

$$
H(0)=\int_{-\infty}^{\infty} h(t) d t .
$$

The path loss of the channel equals the DC gain of the channel $H(0)$ measured in decibels $(\mathrm{dB})$.

The shot noise in (4) accounts for the received power from the intended transmitter and the received interference power form the surrounding vehicles. Therefore, the SNR at the receiver is given by [24]

$$
S N R=\frac{\gamma^{2} P_{R}^{2}}{\sigma_{T}^{2}} .
$$

The on-off keying (OOK) modulation scheme is widely considered as a competent candidate in VVLC systems that use intensity modulation / direct detection (IM/DD) schemes because it is simple and resilient to ambient noise and the nonlinearity distortion of the LED [11], [18]. Therefore, considering OOK modulation scheme, the BER of the system is given by [11]

$$
B E R=Q(\sqrt{S N R})
$$

where

$$
Q(y)=\frac{1}{\sqrt{2 \pi}} \int_{0}^{\infty} e^{-y^{2} / 2} d y .
$$

The equations (5), (7) and (8) show that to establish the communication performance, the DC channel gain is required. In the next section, we present the theoretical study of V2VVLC channel gain.

\section{THE V2V-VLC CHANNEL GAIN}

To characterize the channel gain, we consider the radiation pattern of vehicles headlights. Vehicles use low-beams at high traffic density when the inter-vehicular distance is short. Based on ECE R112 regulation, the radiation pattern of low-beam headlights is asymmetrical, directed towards road surface and 


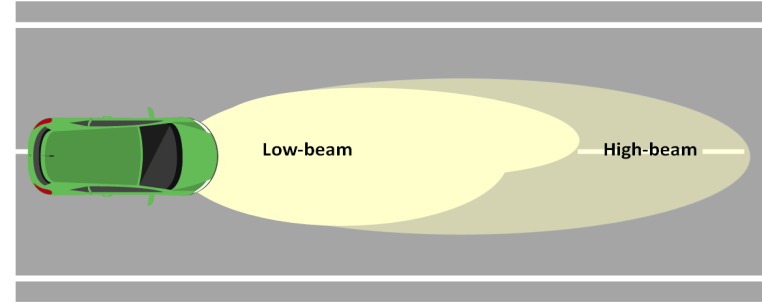

Fig. 2: A schematic (not to scale) of high-beam and low-beam patterns projection on the road surface.

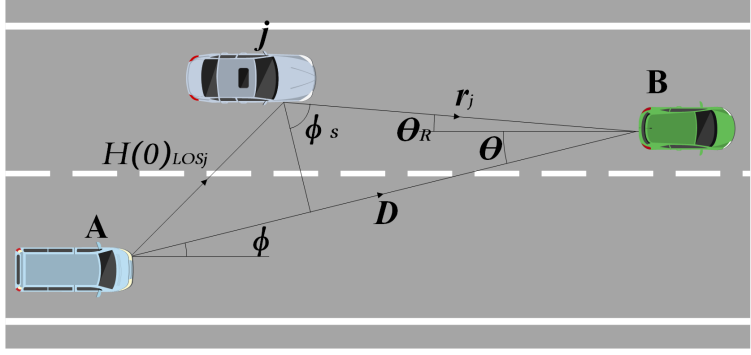

Fig. 3: The LOS and NLOS propagation paths of the optical signal between the transmitter vehicle $\mathrm{A}$ and the receiver vehicle $\mathrm{B}$.

with shorter luminous range to avoid driver blindness by the direct light from vehicles coming from the opposite direction [26]-[28]. At low traffic density when the inter-vehicular distance is longer, vehicles use high-beam headlights. The high-beam headlights radiation pattern is not restricted by the ECE R112 regulation [27]. Therefore, the Lambertian model is considered to describe the symmetrical radiation pattern of high-beam headlights with LED. A schematic of highbeam and low-beam patterns projection on the road surface is illustrated in Fig. 2.

The LOS and NLOS propagation paths of the optical signal between the transmitter vehicle $\mathrm{A}$ and the receiver vehicle $\mathrm{B}$ are illustrated in Fig. 3. The DC channel gain of LOS path for the Lambertian source is given by [28]

$$
H_{L O S}(0)=\frac{A_{r}(m+1)}{2 \pi D^{2}} \cos ^{m}(\phi) \cos (\theta)
$$

where $D$ is the inter-vehicular distance, $\phi$ is the irradiance angle, $\theta$ is the incident angle at the PD, as illustrated in Fig. 3, and $m=\frac{-0.6931}{\ln \left(\cos \left(\Psi_{1 / 2}\right)\right)}$ is the Lambertian order and $\Psi_{1 / 2}$ is the half-power angle of the radiation.

In this study, we use an empirical radiation model of the source as outlined in [29]. This model describes the angular distribution of the asymmetric radiation intensity pattern of the low-beam headlight. Accordingly, the LOS channel path loss (in $\mathrm{dB}$ ) is given by [29]

$P_{L O S}=\alpha+\delta-10 \beta \log _{10}(D+1)+\epsilon \cos \left(\frac{2 \pi(\phi+90)}{\omega}\right)$

where the numerical constant $(\alpha=695.3, \delta=-717.3, \beta=$ 4.949, $\epsilon=63.13$, and $\omega=173$ ) were determined from the empirical measurements of vehicle headlight using the nonlinear least square method [29].

Considering a Lambertian diffuse reflection from vehicle surfaces [30], [31], the channel gain from the reflec-

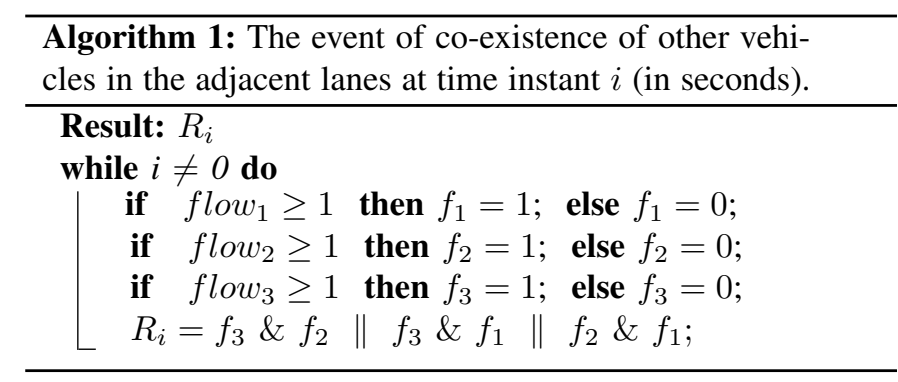

tors is given as

$$
H_{\text {ref }}(0)=\sum_{j=1}^{N} \frac{\rho(m+1) H_{L O S_{j}}(0)}{2 \pi r_{j}^{2}} \cos \left(\phi_{s}^{j}\right) \cos \left(\theta_{R}^{j}\right)
$$

where $H_{L O S j}(0)$ is the LOS DC channel gain from the source to the $j^{t h}$ reflector, as illustrated in Fig. $3, N$ is the total number of reflectors, $r_{j}$ is the distance from the $j^{\text {th }}$ reflector to the receiver, $\rho$ is the reflective index of the reflection surface, $\theta_{R}$ is the incidence angle at the receiver and $\phi_{s}$ is the irradiance angle with respect to the normal to the reflector. The power received from reflections is $P_{R_{\text {ref }}}=P_{T} L H_{\text {ref }}(0)$.

The performance of the V2V-VLC link is affected by the existence of other vehicles in the adjacent lanes, which induce additional noise and multipath propagation. Therefore, in the next section, we study the probability of co-existence of other vehicles in the adjacent lanes.

\section{PROBABILITY OF CO-EXISTENCE OF OTHER VEHICLES IN THE ADJACENT LANES}

In this study, we use traffic measurements to calculate the probability of co-existence of other vehicles in the adjacent lanes. We determine the existence of vehicles from traffic flow and road occupancy data.

To calculate the co-existence of other vehicles in the adjacent lanes, we first define the event $f_{k}$, which indicates that the number of vehicles in the $k^{t h}$ lane $(k=1,2,3$ for the lefthand, middle, and the right-hand lanes, respectively) is larger than one. A logic 1 is assigned to the event $f_{k}$ to indicate that the traffic flow in the $k^{t h}$ lane $\left(\right.$ flow $\left._{k}\right)$ is larger than one, and hence the number of vehicles is larger than one, and 0 otherwise. Then, we calculate the event $R_{i}$ of having at least two vehicles in any two lanes, as described in Algorithm 1.

However, the probability of co-existence of other vehicles in the adjacent lanes is reinforced by the increasing road occupation percentages $O_{k}$ which measures the time duration of a vehicle occupying the $k^{t h}$ lane. Therefore, the probabilities of co-existence of other vehicles in the adjacent lanes $L_{k}$ is given as a product of the event of having two vehicles in any two lanes $R_{i}$ and the percentages that these vehicles occupy the road for a longer time $O_{k}$, which are given by

$$
\begin{gathered}
L_{3}=R_{i} \times\left(\frac{O_{2}+O_{1}}{2}\right) \\
L_{2}=R_{i} \times\left(\frac{O_{3}+O_{1}}{2}\right) \\
L_{1}=R_{i} \times\left(\frac{O_{3}+O_{2}}{2}\right) .
\end{gathered}
$$



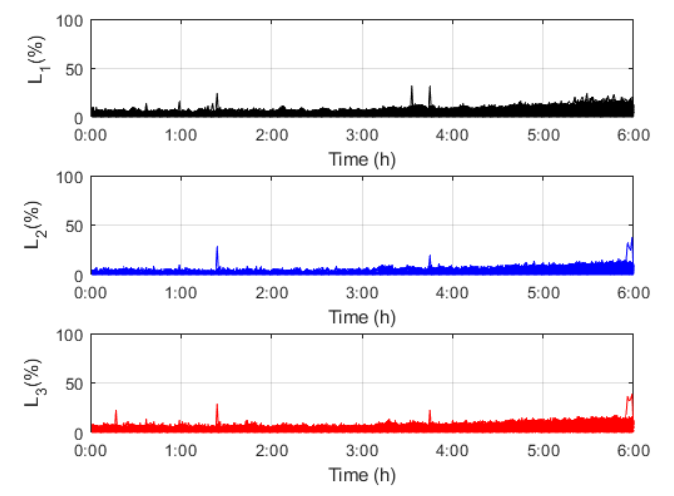

Fig. 4: The probability of co-existence of other vehicles in the adjacent lanes during the late night and early morning hours (00:00-06:00).

Then, the distribution that describes the probability of the co-existence of other vehicles in the adjacent lanes can be calculated using the distribution of $L_{k}$.

\section{Data Processing, Results, and Discussion}

\section{A. Traffic data processing}

Traffic flow and road occupancy were obtained from data collected from the multiple-loop sensors on the M6 and M42 motorways in the UK. The data was collected on the 21, 24, and 28 November 2017 on the M42, and on 6, 7, and 8 December 2017 on the M6. Consecutive 60 minutes of traffic data were collected over 24 hours period starting at midnight. The measurements for the M42 and M6 were collected using 318 and 154 sensors, respectively. The M42 is a smart motorway with enforced variable speed limits and hard shoulder running at rush hours, whereas the M6 was, at the time the data were collected, a standard motorway. However, in our analysis, we considered three lanes excluding the hard shoulder lane. We did not observe a difference between the statistics of both motorways at this particular data set. Therefore, the resulting models were averaged to improve the model generalisability. The traffic flow and road occupancy per lane are measured when vehicles enter and exit the sensor loop on the road.

In order to study the statistics of the event of the coexistence of other vehicles in the adjacent lanes during different periods of time, we analyse the probability of the co-existence of other vehicles in the adjacent lanes versus time. We consider the traffic during a day in three lanes; the right-hand lane (Lane 3), middle lane (Lane 2), and lefthand lane (Lane 1). According to the traffic density, we consider three periods 00:00-06:00, 06:00-20:00, and 20:00-24:00. The time periods 00:00-06:00, and 20:00-24:00 have low traffic density. The time period 06:00-20:00 has high traffic density. Depending on the analysis described in section IV, the probability of the co-existence of other vehicles in the adjacent lanes $L_{k}$ during the low-density traffic during the late night and early morning hours from 00:00 to 06:00 is presented in Fig. 4. The figure shows that the probabilities of the co-existence of other vehicles in the adjacent lanes are initially below $10 \%$, in the three lanes. Then, it starts to increase gradually after 03:00, exceeding 20\%. Therefore, we
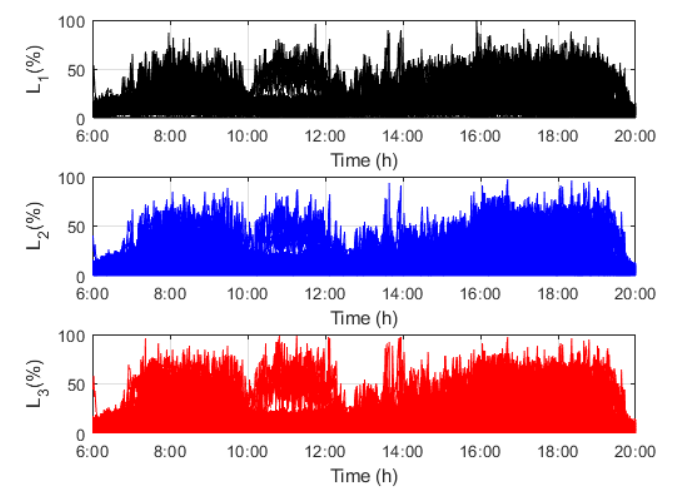

Fig. 5: The probability of co-existence of other vehicles in the adjacent lanes during the rush hours (06:00-20:00).
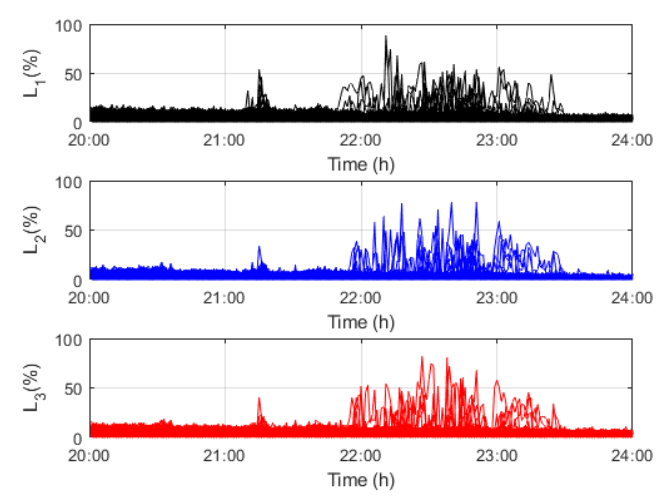

Fig. 6: The probability of co-existence of other vehicles in the adjacent lanes during the night hours (20:00-24:00).

use the time window between 00:00 and 03:00 to study the off-peak probability of the co-existence of other vehicles in the adjacent lanes in the rest of this study.

The probability of the co-existence of other vehicles in the adjacent lanes during the rush hours (06:00-20:00) is presented in Fig. 5. The figure shows that the probability of the coexistence of other vehicles in the adjacent lanes increases significantly $(>60 \%)$ after 7:00. These high probability values can be noticed over the entire time window. It can reach $90 \%$ between 16:00 and 19:00. Therefore, we use this time window to study the probabilities of the co-existence of other vehicles in the adjacent lanes when the traffic density has the highest values.

The probability of the co-existence of other vehicles in the adjacent lanes during the night hours (20:00-24:00) is presented in Fig. 6. The figure shows that the probability of the co-existence of other vehicles in the adjacent lanes below 10\% between 20:00 and 22:00, for the three lanes. During the time window 22:00-23:00, the probability of the coexistence of other vehicles in the adjacent lanes increases, but it does not exceed $60 \%$. The probability of the co-existence of other vehicles decreases again to below $10 \%$ at the last hour of the day.

To establish the distribution that closely estimates coexistence of other vehicles in the adjacent lanes, we performed a cumulative distribution function (CDF) curve fitting method with Normal, log-normal, exponential, and Nakagami distri- 
TABLE I: Parameters of different distributions that are expected to describes $L$.

\begin{tabular}{|c|c|c|c|c|c|c|c|}
\hline Time & Lane & Distribution & Mean Value (m) & $\mu_{l}$ & $\mu_{l}$ estimation error (\%) & $\delta_{l}$ & $\delta_{l}$ estimation error(\%) \\
\hline \multirow{12}{*}{ 00:00-03:00 } & left (1) & \multirow{3}{*}{ log-normal } & 3.74 & 1.21 & 0.41 & 0.46 & 0.29 \\
\hline & middle (2) & & 2.55 & 0.78 & 0.98 & 0.56 & 0.69 \\
\hline & right (3) & & 3.61 & 1.18 & 0.34 & 0.45 & 0.24 \\
\hline & left (1) & \multirow{3}{*}{ Nakagami } & 3.7 & 1.56 & 1.8 & 16.2 & 11.67 \\
\hline & middle (2) & & 2.6 & 0.94 & 2.0 & 8.7 & 15.7 \\
\hline & right (3) & & 3.6 & 1.6 & 1.6 & 15.1 & 9.3 \\
\hline & left (1) & \multirow{3}{*}{ Normal } & 3.7 & 3.7 & 1.4 & 1.6 & 1.0 \\
\hline & middle (2) & & 2.5 & 2.5 & 2.6 & 1.5 & 1.8 \\
\hline & right (3) & & 3.6 & 3.6 & 1.2 & 1.5 & 0.8 \\
\hline & left (1) & \multirow{3}{*}{ Exponential } & 3.7 & 3.7 & 3.3 & - & - \\
\hline & middle (2) & & 2.5 & 2.5 & 4.4 & - & - \\
\hline & right (3) & & 3.6 & 3.6 & 2.8 & - & - \\
\hline \multirow{12}{*}{$16: 00-19: 00$} & left-hand (1) & \multirow{3}{*}{ log-normal } & 19.9 & 2.71 & 0.30 & 0.70 & 0.25 \\
\hline & middle (2) & & 16.7 & 2.44 & 0.35 & 0.87 & 0.20 \\
\hline & right (3) & & 17.4 & 2.58 & 0.45 & 0.74 & 0.21 \\
\hline & left (1) & \multirow{3}{*}{ Nakagami } & 19.3 & 0.73 & 0.38 & 515 & 262 \\
\hline & middle (2) & & 15.9 & 0.58 & 0.28 & 380 & 205 \\
\hline & right (3) & & 17.8 & 0.64 & 0.31 & 457 & 235 \\
\hline & left (1) & \multirow{3}{*}{ Normal } & 18.9 & 18.9 & 5.4 & 12.5 & 3.8 \\
\hline & middle (2) & & 15.6 & 15.6 & 4.5 & 11.7 & 3.9 \\
\hline & right (3) & & 17.1 & 17.1 & 5.2 & 12.7 & 3.7 \\
\hline & left (1) & \multirow{3}{*}{ Exponential } & 18.9 & 18.9 & 8.27 & - & - \\
\hline & middle (2) & & 15.6 & 15.6 & 6.4 & - & - \\
\hline & right (3) & & 17.2 & 17.2 & 7.0 & - & - \\
\hline
\end{tabular}

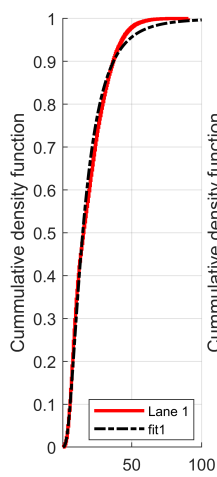

$\mathrm{L}(\%)$

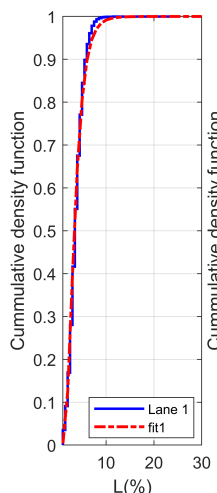

$\mathrm{L}(\%)$

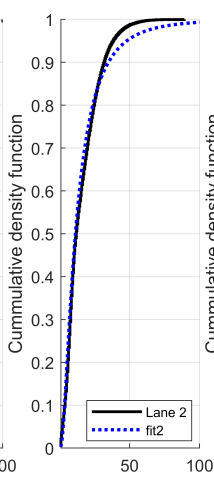

$\mathrm{L}(\%)$

(a)

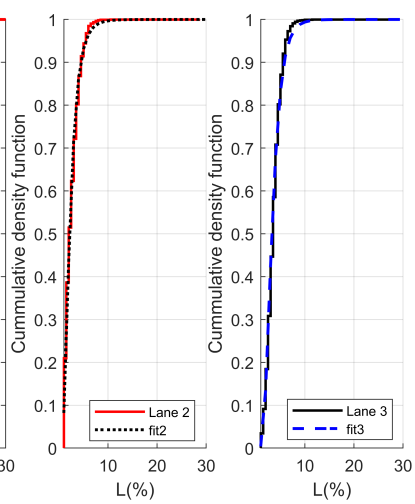

(b)

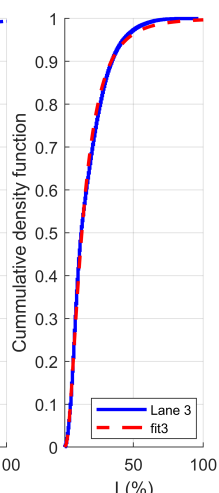

L(\%)$$
\text { . }
$$ 
TABLE II: Parameters of different distributions that are expected to describe $D$.

\begin{tabular}{|c|c|c|c|c|c|c|c|}
\hline Time & Lane & Distribution & Mean Value (m) & $\mu_{d}$ & $\mu_{d}$ estimation error (\%) & $\delta_{d}$ & $\delta_{d}$ estimation error(\%) \\
\hline \multirow{12}{*}{ 00:00-03:00 } & left (1) & \multirow{3}{*}{ log-normal } & 57.2 & 3.72 & 0.45 & 0.80 & 0.32 \\
\hline & middle (2) & & 67.1 & 3.82 & 0.44 & 0.87 & 0.31 \\
\hline & right (3) & & 109.8 & 4.35 & 0.64 & 0.83 & 0.45 \\
\hline & left (1) & \multirow{3}{*}{ Nakagami } & 62.1 & 0.5 & 0.33 & 6032 & 4844 \\
\hline & middle (2) & & 73.3 & 0.45 & 0.26 & 8770 & 6630 \\
\hline & right (3) & & 107.9 & 0.59 & 0.54 & 17131 & 1723 \\
\hline & left (1) & \multirow{3}{*}{ Normal } & 57.2 & 57.2 & 29.8 & 52.3 & 21.0 \\
\hline & middle (2) & & 67.1 & 67.1 & 33.2 & 65.3 & 23.4 \\
\hline & right (3) & & 104.0 & 104.0 & 78.9 & 61.2 & 43.3 \\
\hline & left (1) & \multirow{3}{*}{ Exponential } & 57.5 & 57.5 & 32.7 & - & - \\
\hline & middle (2) & & 67.1 & 67.1 & 34.1 & - & - \\
\hline & right (3) & & 104.0 & 104.0 & 81.0 & - & - \\
\hline \multirow{12}{*}{$16: 00-19: 00$} & left (1) & \multirow{3}{*}{ log-normal } & 7.8 & 1.35 & 0.45 & 1.18 & 0.31 \\
\hline & middle (2) & & 3.9 & 0.81 & 0.42 & 1.05 & 0.29 \\
\hline & right (3) & & 2.5 & 0.50 & 0.27 & 0.90 & 0.39 \\
\hline & left (1) & \multirow{3}{*}{ Nakagami } & 11.9 & 0.2 & 0.10 & 325 & 256 \\
\hline & middle (2) & & 8.3 & 0.2 & 0.09 & 169 & 148 \\
\hline & right (3) & & 5.2 & 0.2 & 0.11 & 63.3 & 57.7 \\
\hline & left (1) & \multirow{3}{*}{ Normal } & 8.6 & 8.6 & 6.0 & 15.8 & 4.2 \\
\hline & middle (2) & & 5.1 & 5.1 & 4.8 & 11.9 & 3.4 \\
\hline & right (3) & & 3.1 & 3.1 & 6.0 & 7.3 & 2.2 \\
\hline & left (1) & \multirow{3}{*}{ Exponential } & 8.6 & 8.6 & 3.27 & - & - \\
\hline & middle (2) & & 5.1 & 5.1 & 2.0 & - & - \\
\hline & right (3) & & 3.1 & 3.1 & 1.3 & - & - \\
\hline
\end{tabular}

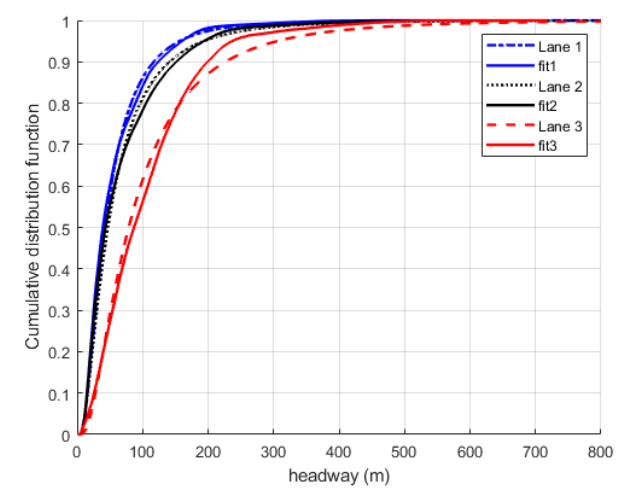

(a)

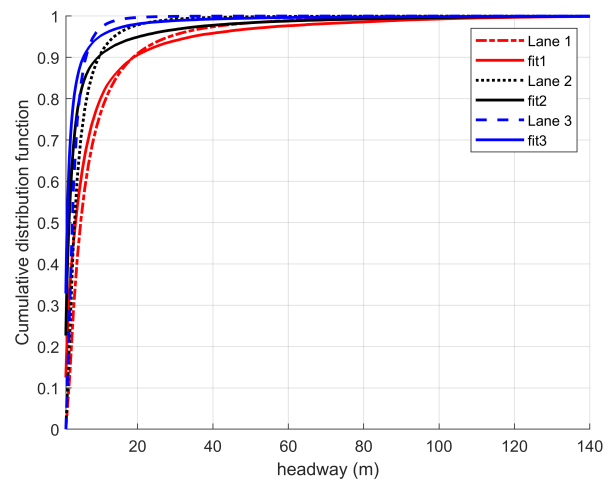

(b)

Fig. 8: Inter-vehicular distribution in three lanes at a) 00:0003:00 and b) 16:00-19:00.

standard error values for these distributions for the three lanes during 00:00-03:00 and 16:00-19:00 hours. The table shows that the log-normal distribution has the least estimation error value. This is consistent with the literature which reported that the log-normal distribution offers a close fit to the intervehicle distance [32]-[34]. Hence, the distribution of the inter- vehicular distances is given by

$$
P_{D}(d)=\frac{1}{\delta_{d} \sqrt{2 \pi}} \frac{1}{d} \exp \left(-\frac{\left(\ln (d)-\mu_{d}\right)^{2}}{2 \delta_{d}^{2}}\right) .
$$

The distribution parameters $\mu_{d}$ and $\delta_{d}$ are given in Table II. The CDFs of the inter-vehicular distances and a log-normal distribution for the three lanes during 00:00-03:00 and 16:0019:00 are depicted in Fig. 8.

\section{B. V2V-VLC link performance results}

The channel quality metrics SNR and the corresponding BER performance of the V2V-VLC link was simulated using the parameters summarized in Table III. The BER performance is evaluated with regard to the transmit SNR to examine the impact of channel path loss variation during different periods of the day. Evaluating system performance in terms of transmit SNR is a standard method in VLC especially when the channel impulse response is varying [35]-[38]. Considering received power in (5) and the SNR at the receiver in (7), the transmit SNR is given by

$S N R_{T}[d B]=10 \log \left(\frac{\gamma^{2} H(0)^{2} P_{T}^{2}}{\sigma_{T}^{2}}\right)-10 \log \left(H(0)^{2}\right)$.

Therefore, the SNR values (in $\mathrm{dB}$ ) at the receiver are offset by the corresponding channel path loss values [35]. The simulation is carried out for a transmitter vehicle and a receiver vehicle separated by an inter-vehicular distance $D$ with a mean value that changes according to transmitter-receiver vehicles' positions on different lanes and during different periods of the day (see Table II). The communication link is affected by the existence of three (i.e., $j=1,2$, and 3) potential reflectors/interferers vehicles on the adjacent lanes located at $\left(x_{j}, y_{j}\right)$ of $(1, D / 3) \mathrm{m},(0.5, D / 2) \mathrm{m}$, and $(2,2 D / 3) \mathrm{m}$ as illustrated in Fig. 1b. However, demand from different lane can be considered by varying the angles $\phi, \theta, \phi_{s}$, and $\theta_{R}$ in (10) and (12) to study other scenarios, as shown in Fig. 3. 
TABLE III: Simulation parameters.

\begin{tabular}{|c|c|c|}
\hline Symbol & Parameter & Values \\
\hline$P_{T}$ & Transmitted power (normalized) & $1 \mathrm{~W}$ \\
\hline$\Phi_{c}$ & Semi-angle & $30^{\circ}[23]$ \\
\hline$\gamma$ & PD responsivity & $0.54 \mathrm{~A} / \mathrm{W}[39]$ \\
\hline$\Psi_{c}$ & The receiver field of view & $80^{\circ}[3]$ \\
\hline$A_{r}$ & Receiver area & $1 \times 10^{-4} \mathrm{~m}^{2}[3]$ \\
\hline$C_{p d}$ & Capacitance of PD per unit area & $1.12 \mu \mathrm{Fm}^{-2}[18]$ \\
\hline$I_{2}$ & Noise bandwidth factor & $0.562[18]$ \\
\hline$I_{3}$ & Noise bandwidth factor & $0.0868[11]$ \\
\hline$B_{w}$ & Noise bandwidth & $100 \mathrm{MHz}[11]$ \\
\hline$G_{\nu}$ & Open-loop voltage gain & $10[18]$ \\
\hline$\Gamma$ & FET channel noise factor & $1.5[18]$ \\
\hline$g_{m}$ & FET transconductance & $30 \mathrm{mS} \mathrm{[18]}$ \\
\hline$I_{b g}$ & Background current at $\lambda=850 \mathrm{~nm}$ & $55.4 \mu \mathrm{A}[11]$ \\
\hline$\rho$ & Reflection coefficient & $0.8[3]$ \\
\hline
\end{tabular}

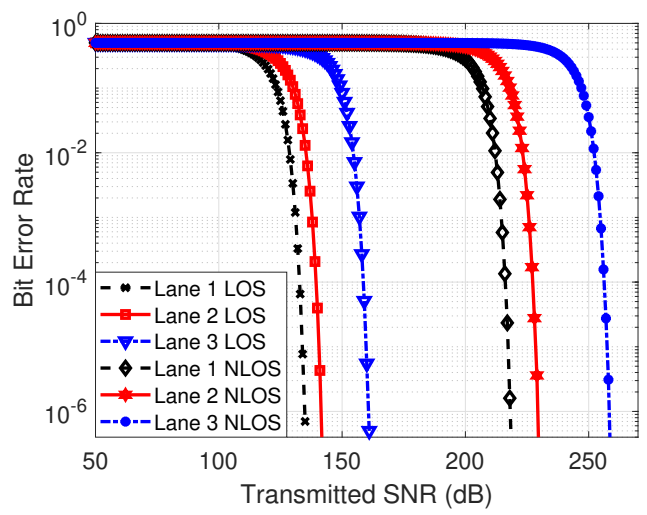

(a)

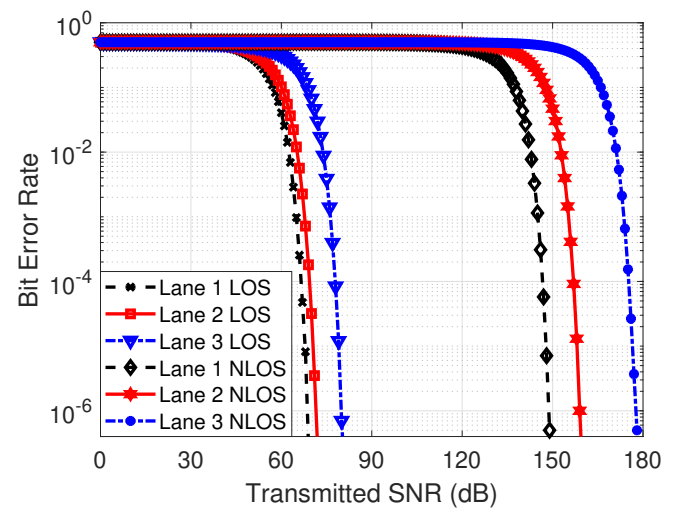

(b)

Fig. 9: BER performance of the V2V-VLC link in three lanes at 00:00-03:00 when a) low-beam and b) high-beam radiation patterns are considered.

BER performance of the V2V-VLC link in each of the three lanes between 00:00 and 03:00 is shown in Fig. 9, where Fig. 9a represents the asymmetrical radiation pattern of the low-beam and Fig. 9b represents the symmetrical radiation pattern of the high-beam headlights. The BER performance is estimated using a pseudorandom sequence of $10^{7}$ bits length and a transmission rate of $50 \mathrm{Mbps}$ assuming OOK modulation. The figures illustrate the BER performance of
LOS components and NLOS components (i.e., excluding the LOS signal) which are reflected from the vehicles in the adjacent lanes. The figures show a large variation of the BER performance over different lanes. This is excepted as the transmission distance in different lanes has different lanedependent mean values as summarised in Table II. During the off-peak hours 00:00-03:00, the left-hand lane (Lane 1) has the shortest transmission distance with a mean value of $57.2 \mathrm{~m}$ compared to the mean values of the inter-vehicular distances of $67.1 \mathrm{~m}$ and $109.8 \mathrm{~m}$ for the middle lane (Lane 2) and the right-hand lane (Lane 3), respectively. Hence, the lefthand lane (Lane 1) has the least path loss and the highest SNR. Therefore, the BER values on this lane are lower than the middle and right-hand lanes (Lane 2 and 3). For example, at SNR $=130 \mathrm{~dB}$, the BER values of the LOS link are $0.452,0.101$, and 0.003 for right-hand lanes (Lane 3), middle lane (Lane 2), and the left-hand lane (Lane 1), respectively. Furthermore, the BER values of the NLOS component for lefthand lane (Lane 1) is lower than the middle and right-hand lanes (Lane 2 and 3), e.g. at $\mathrm{SNR}=205 \mathrm{~dB}$, the BER values of the NLOS links are $0.49,0.38$, and 0.07 for right-hand lanes (Lane 3), middle lane (Lane 2), and the left-hand lane (Lane 1), respectively. Fig. 9a shows that at $\mathrm{SNR}=60 \mathrm{~dB}$, the BER values of the LOS links are 0.31, 0.10, and 0.04 for the right-hand lane (Lane 3), middle lane (Lane 2), and left-hand lanes (Lane 1), respectively. Hence, the V2V-VLC system is practically feasible when the high-beam is used. The figures show a large difference between the BER performance of the LOS component and the NLOS component as it is expected in Table I because the mean value of the probability of the co-existence of other vehicles in the adjacent lanes does not exceed $3.74 \%$, i.e., the probability of reflection occurrence is very low.

BER performance of the V2V-VLC link in the three lanes at rush hours (16:00-19:00) is shown in Fig. 10. The figure shows that the BER performance of right-hand lane (Lane 3) is better than the middle and left-hand lanes (Lane 2 and 1). For example, at SNR $=10 \mathrm{~dB}$, the BER values are 0.489, 0.290, and 0.001 for left-hand lanes (Lane 1), middle lane (Lane 2), and the right-hand lane (Lane 3), respectively. This is expected as the right-hand lane (Lane 3) has the shortest inter-vehicular distance with a mean value of $2.48 \mathrm{~m}$ during rush hours compared to the mean values of $3.93 \mathrm{~m}$, and $7.79 \mathrm{~m}$ for middle lane (Lane 2), and the lefthand lane (Lane 1), respectively (see Table II). The BER values of the NLOS and NLOS links are close for the right-hand lane (Lane 3). The difference in the BER performance of the NLOS and the LOS links increases in the middle lane (Lane 2) and the left-hand lane (Lane 1). The adequate performance of NLOS component is also expected from Section V.A due to the high probability of the co-existence of other vehicles in the adjacent lanes which can reach $90 \%$, i.e., the probability of reflection occurrence is high.

Fig. 9 and Fig. 10 also show that the required SNR ranges vary widely from hundreds of decibels during the off-peak hours to tens of decibels during the rush hours to achieve comparable BER values. This indicates the variation and dependency of channel capacity on the traffic conditions during 


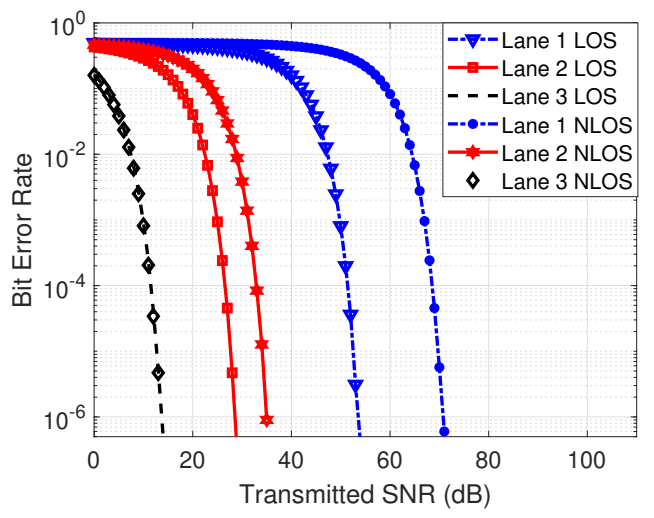

Fig. 10: BER performance of the V2V-VLC link in three lanes at 16:00-19:00 when lo-beam radiation pattern is considered.

different periods of the day. In addition, the figures examined the BER performance of the V2V-VLC system for a range of the transmitted SNR that depends on the illumination level requirements. In [35]-[38], the transmitted SNR values ranged from $80 \mathrm{~dB}$ to $240 \mathrm{~dB}$ for an indoor environment when the transmission distance does not exceed $5 \mathrm{~m}$. The actual SNR depends on a number of factors such transmitter and receiver technology, data rate, optical gain and optical filters. However, to fulfil illumination constraints, the transmitted optical power is a constant i.e. the transmitter SNR is fixed for a given system. Consequently, depending upon the transmitter SNR, the BER values change. This is a common challenge in mobile communication links. To ensure a minimum of communication requirements, various techniques such as automatic gain control, adaptive data rate and rate-adaptive modulation are used [40], [41].

Table IV provides a summary of the required SNR (at the transmitter) values to achieve a BER of $10^{-6}$ for different lamps, lanes, and propagation paths. The table shows that higher SNR values are required to achieve acceptable BER performance at off-peak hours regardless of the propagation path (i.e., LOS or NLOS) compared to the rush hours. This is because the average inter-vehicular distance is lower during the rush hours compared to the late hours. The table also shows that communication using the NLOS path is feasible during rush hour. This is an important observation as the blocking probability of the LOS is significantly higher during the rush hours. However, the V2V-VLC is still feasible with only NLOS. In late hours, the blocking probability is less and LOS is available. This indicates that V2V-VLC is available for both rush and off-peak hours. Furthermore, for the same channel condition, the SNR requirements for the high-beam radiation are lower than the low-beam. This is due to the directional radiation pattern of high-beam which reduces the path loss.

\section{CONCLusions}

This paper presented the impact of dynamic traffic on the performance of V2V-VLC for different lanes on two UK motorways at different times of the day. Using traffic measurements, the average inter-vehicular distances were estimated and the BER performance was evaluated. The inter-vehicular
TABLE IV: summary of the required SNR values to achieve $\mathrm{BER}=10^{-6}$ for different lamps, lanes, and propagation path, when OOK modulation scheme is considered.

\begin{tabular}{|c|c|c|c|c|c|c|c|}
\hline Time & \multicolumn{3}{c|}{$00: 00-03: 00$} & \multicolumn{2}{c|}{ 16:00-19:00 } \\
\hline \multicolumn{2}{|c|}{ Lamp } & \multicolumn{2}{c|}{ high-beam } & \multicolumn{2}{c|}{ low-beam } & \multicolumn{2}{c|}{ low-beam } \\
\hline \multicolumn{2}{|c|}{ Path } & LOS & NLOS & LOS & NLOS & LOS & NLOS \\
\hline \multirow{2}{*}{$\begin{array}{c}\text { SNR } \\
\text { (dB) }\end{array}$} & left (1) & 68.6 & 148.7 & 134.8 & 218.2 & 53.6 & 70.8 \\
\cline { 2 - 8 } & middle (2) & 71.5 & 159.0 & 141.5 & 229.3 & 28.6 & 35.0 \\
\cline { 2 - 8 } & right (1) & 79.9 & 177.7 & 160.7 & 258.3 & 13.7 & 13.5 \\
\hline
\end{tabular}

distance depends on the traffic conditions as well as on the lane. Therefore, the BER performance at different lanes was evaluated for different traffic conditions and lanes. During offpeak hours, the BER at the left-hand lane is the lowest compared to the middle and right-hand lanes. This is because the mean inter-vehicular distance in the left-hand lane is $57.2 \mathrm{~m}$ which is lower than $67.1 \mathrm{~m}$ and $109.8 \mathrm{~m}$ for the middle and right-hand lanes, respectively. During the rush hours, the righthand lane has lower BER values than those of the middle and the left-hand lanes because the inter-vehicular distance in this lane has a lower mean value of $2.48 \mathrm{~m}$ compared to $3.93 \mathrm{~m}$ and $7.79 \mathrm{~m}$ for the middle and left-hand lanes, respectively. Considering a large variation of inter-vehicular distances between rush and off-peak hours, lower values of SNR are required during rush hours to achieve comparable BER performance e.g. at a BER of $10^{-6}$ the SNR required at right-hand lane for LOS links are $13.7 \mathrm{~dB}$ and $160.7 \mathrm{~dB}$ at the rush and off-peak hours, respectively.

In addition, the study adopted a statistical modelling approach to model the probability of co-existence of other vehicles in adjacent lanes and the impact of reflection from these vehicles. The results revealed that the probability of co-existence of other vehicles in the adjacent lanes during the rush hours is as high as $90 \%$, while it decays to less than $10 \%$ during off-peak traffic hours. Hence, the probability of reflection from the adjacent vehicles is high during rush hours. Therefore, the performance of NLOS components due to reflection is significant and comparable to the LOS component, i.e., SNR values of less than $70 \mathrm{~dB}$ are required to achieve a BER value of $10^{-6}$. However, the performance of NLOS components is insignificant during off-peak hours as the required SNR values to achieve BER $=10^{-6}$ are larger than $100 \mathrm{~dB}$.

\section{ACKNOWLEDGEMENTS}

This work was supported by the innovate-UK Project Park-IT (Reference: 10509)(partially), the National Key R\&D Program of China under Grant 2020YFB1804901, the National Natural Science Foundation of China (NSFC) under Grant 61960206006, and the EU H2020 RISE TESTBED2 project under Grant 872172. The authors would like to thank Highways England and Mott MacDonald for providing us with traffic data.

\section{REFERENCES}

[1] World Health Organization, "Global status report on road safety 2018," no. 9789241565684, 2018. [Online]. Available: https://www.who.int/publications/i/item/9789241565684

[2] S. Rehman, S. Ullah, P. Chong, S. Yongchareon, and D. Komosny, "Visible light communication: a system perspective-overview and challenges," Sensors, vol. 19, no. 5, p. 1153, Mar. 2019. 
[3] A. Al-Kinani, J. Sun, C. Wang, W. Zhang, X. Ge, and H. Haas, "A 2-D non-stationary GBSM for vehicular visible light communication channels," IEEE Trans. Wireless Commun., vol. 17, no. 12, pp. 79817992, Dec. 2018.

[4] C. X. Wang, J. Huang, H. Wang, X. Gao, X. You, and Y. Hao, "6G wireless channel measurements and models: trends and challenges," IEEE Veh. Technol. Mag., vol. 15, no. 4, pp. 22-32, 2020.

[5] Q. Chen, C. X. Wang, J. Sun, W. Zhang, and Q. Zhu, "A non-stationary VVLC MIMO channel model for street corner scenarios," in Proc. 2020 Int. Wireless Commun. and Mobile Comput. (IWCMC'20), Limassol, Cyprus, July 2020, pp. 365-370.

[6] X. You, C.-X. Wang, J. Huang, X. Gao, Z. Zhang, M. Wang, Y. Huang, C. Zhang, Y. Jiang, J. Wang et al., "Towards 6G wireless communication networks: vision, enabling technologies, and new paradigm shifts," Sci. China Inf. Sci., vol. 64, no. 1, pp. 1-74, Jan. 2021.

[7] M. Uysal, Z. Ghassemlooy, A. Bekkali, A. Kadri, and H. Menouar, "Visible light communication for vehicular networking: Performance study of a V2V system using a measured headlamp beam pattern model," IEEE Veh. Technol. Mag., vol. 10, no. 4, pp. 45-53, Dec. 2015.

[8] B. Béchadergue, L. Chassagne, and H. Guan, "Simultaneous visible light communication and distance measurement based on the automotive lighting," IEEE Trans. Intell. Veh., vol. 4, no. 4, pp. 532-547, Aug. 2019.

[9] J. Chen and Z. Wang, "Topology control in hybrid VLC/RF vehicular Ad-Hoc network," IEEE Trans. Wireless Commun., vol. 19, no. 3, pp. 1965-1976, Mar. 2020.

[10] L. U. Khan, "Visible light communication: Applications, architecture, standardization and research challenges," Digit. Commun. Netw., vol. 3, no. 2, pp. 78-88, May. 2017

[11] Z. Ghassemlooy, W. Popoola, and S. Rajbhandari, Optical wireless communications: system and channel modelling with Matlab ${ }^{\circledast}$. Boca Raton, FL: CRC Press (Inc.), 2019.

[12] G. Singh, A. Srivastava, and V. A. Bohara, "Impact of weather conditions and interference on the performance of VLC based V2V communication," in Proc. Int. Conf. Transparent Opt. Netw. (ICTON'19), Angers, France, Sep. 2019, pp. 1-4.

[13] I. E. Lee, M. L. Sim, and F. W. L. Kung, "Performance enhancement of outdoor visible-light communication system using selective combining receiver," IET Optoelectronics, vol. 3, no. 1, pp. 30-39, Feb. 2009.

[14] M. S. Islim, S. Videv, M. Safari, E. Xie, J. J. D. McKendry, E. Gu, M. D Dawson, and H. Haas, "The impact of solar irradiance on visible light communications," J. Lightw. Technol., vol. 36, no. 12, pp. 2376-2386, Mar. 2018.

[15] Y. H. Chung and S. Oh, "Efficient optical filtering for outdoor visible light communications in the presence of sunlight or artificial light," in Proc. 2013 Int. Symp. Intell. Signal Process. Commun. Syst. (ISISPCS'13), Naha, Japan, Nov. 2013, pp. 749-752.

[16] D. Cervinka, Z. Ahmad, O. Salih, and S. Rajbhandari, "A study of yearly sunlight variance effect on vehicular visible light communication for emergency service vehicles," in Proc. 12th IEEE/IET Int. Symp. Commun. Syst. Netw. and Digit. Signal Process. (CSNDSP'20), Porto, Portugal, July 2020, pp. 1-6.

[17] R. Khoder, R. Naja, and S. Tohme, "Impact of interference on visible light communication performance in a vehicular platoon," in Proc. 2020 Int. Wireless Commun. Mobile Comput. (IWCMC'20), Limassol, Cyprus, July 2020, pp. 1935-1939.

[18] P. Luo, Z. Ghassemlooy, H. L. Minh, E. Bentley, A. Burton, and X. Tang, "Performance analysis of a car-to-car visible light communication system," Appl. Opt., vol. 54, no. 7, pp. 1696-1706, Mar. 2015.

[19] P. Luo, Z. Ghassemlooy, E. Bentley, A. Burton, and X. Tang, "Biterror-rate performance of a car-to-car VLC system using $2 \times 2$ MIMO," Mediterranean J. Comput. Netw., vol. 11, pp. 400-407, Jan. 2015.

[20] A. Al-Kinani, C. Wang, Q. Zhu, Y. Fu, E. M. Aggoune, A. Talib, and N. A. Al-Hasaani, "A 3D non-stationary GBSM for vehicular visible light communication MISO channels," IEEE Access, vol. 8, pp. 140333 140347 , July 2020.

[21] F. M. Alsalami, Z. Ahmad, O. Haas, and S. Rajbhandari, "Regularshaped geometry-based stochastic model for vehicle-to-vehicle visible light communication channel," in Proc. 2019 IEEE Jordan Int. Joint Conf. Elect. Eng. Inf. Technol. (JEEIT'19), Amman, Jordan, Apr. 2019, pp. 297-301.

[22] F. M. Alsalami, N. Aigoro, A. Mahmoud, Z. Ahmad, P. A. Haigh, O. C. Haas, and S. Rajbhandari, "Impact of vehicle headlights radiation pattern on dynamic vehicular visible light communication system channel," J. Lightw. Technol., vol. Early Access, pp. 1-1, 2021.

[23] F. M. Alsalami, Z. Ahmad, S. Zvanovec, P. A. Haigh, O. C Haas, and S. Rajbhandari, "Statistical channel modelling of dynamic vehicular visible light communication system," Veh.
Commun., vol. 29, p. 100339, June 2021. [Online]. Available: https://www.sciencedirect.com/science/article/pii/S2214209621000085

[24] M. Rahaim and T. D. C. Little, "Interference in IM/DD optical wireless communication networks," IEEE/OSA J. Opt. Commun. Netw., vol. 9, no. 9, pp. D51-D63, Sep. 2017.

[25] M. A. Esmail, H. Fathallah, and M. Alouini, "Outage probability analysis of FSO links over foggy channel," IEEE Photon. J., vol. 9, no. 2, pp. 1-12, Apr. 2017.

[26] F. Chen, K. Wang, Z. Qin, D. Wu, X. Luo, and S. Liu, "Design method of high-efficient LED headlamp lens," Opt. Exp., vol. 18, no. 20, pp. 20 926-38, Sep 2010.

[27] United Nations, "United Nations Economic Commission for Europe vehicle regulations, Reg. 112-Rev.3," UN, no. 9789241565684, Jan. 2013. [Online]. Available: https://www.unece.org/fileadmin/DAM/trans/main/wp29/wp29regs/2013 /R112r3e.pdf

[28] L. Cheng, W. Viriyasitavat, M. Boban, and H. Tsai, "Comparison of radio frequency and visible light propagation channels for vehicular communications," IEEE Access, vol. 6, pp. 2634-2644, Dec. 2018.

[29] A. Memedi, H. Tsai, and F. Dressler, "Impact of realistic light radiation pattern on vehicular visible light communication," in Proc. 2017 IEEE Global Commun. Conf. (GLOBECOM'17), Singapore, Dec. 2017, pp. $1-6$.

[30] N. K. Akafuah, S. Poozesh, A. Salaimeh, G. Patrick, K. Lawler, and K. Saito, "Evolution of the automotive body coating process-a review," Coatings, vol. 6, no. 2, pp. 1-24, June 2016.

[31] R. Ďurikovič and T. Ágošton, "Prediction of optical properties of paints," Central Eur. J. Phys., vol. 5, no. 3, pp. 416-427, Sep 2007.

[32] I. Greenberg, "The log-normal distribution of headways," Australian Road Res., vol. 2, no. 7, Mar. 1966.

[33] G. Yan and S. Olariu, "A probabilistic analysis of link duration in vehicular Ad Hoc networks," IEEE Trans. Intell. Transp. Syst., vol. 12, no. 4, pp. 1227-1236, Dec. 2011.

[34] R. Roy and P. Saha, "Headway distribution models of two-lane roads under mixed traffic conditions: a case study from india," Eur. Transp. Res. Rev., vol. 10, no. 1, pp. 1-12, Mar. 2018.

[35] H. Marshoud, P. C. Sofotasios, S. Muhaidat, G. K. Karagiannidis, and B. S. Sharif, "On the performance of visible light communication systems with non-orthogonal multiple access," IEEE Trans. Wireless Commun., vol. 16, no. 10, pp. 6350-6364, July 2017.

[36] P. M. Butala, H. Elgala, and T. D. C. Little, "Performance of optical spatial modulation and spatial multiplexing with imaging receiver," in Proc. Wireless Commun. Netw. Conf. (WCNC'14), Istanbul, Turkey, Nov. 2014, pp. 394-399.

[37] C. Chen, H. Yang, P. Du, W. D. Zhong, A. Alphones, Y. Yang, and X. Deng, "User-centric MIMO techniques for indoor visible light communication systems," IEEE Syst. J., vol. 14, no. 3, pp. 3202-3213, Jan. 2020.

[38] C. He, T. Q. Wang, and J. Armstrong, "Performance comparison between spatial multiplexing and spatial modulation in indoor MIMO visible light communication systems," in Proc. 2016 IEEE Int. Conf. Communi. (ICC'16), 2016, pp. 1-6.

[39] Y. H. Kim, W. A. Cahyadi, and Y. H. Chung, "Experimental demonstration of VLC-based vehicle-to-vehicle communications under fog conditions," IEEE Photon. J., vol. 7, no. 6, pp. 1-9, Dec. 2015

[40] A.-M. Căilean, M. Dimian, and V. Popa, "Noise-adaptive visible light communications receiver for automotive applications: a step toward selfawareness," Sensors, vol. 20, no. 13, p. 3764, 2020

[41] A. Căilean, M. Dimian, V. Popa, L. Chassagne, and B. Cagneau, "Novel DSP receiver architecture for multi-channel visible light communications in automotive applications," IEEE Sensors Journal, vol. 16, no. 10, pp. $3597-3602,2016$. 